\title{
Robust Multiresolution Techniques for Image Reconstruction
}

\author{
Michael Liebling \\ Biological Imaging Center, Beckman Institute, California Institute of Technology, \\ Mail Code 139-74, Pasadena, CA 91125, USA
}

\begin{abstract}
The reconstruction of images from projections, diffraction fields, or other similar measurements requires applying signal processing techniques within a physical context. Although modeling of the acquisition procedure can conveniently be carried out in the continuous domain, actual reconstruction from experimental measurements requires the derivation of discrete algorithms that are accurate, efficient, and robust. In recent years, wavelets and multiresolution approaches have been applied successfully for common image processing tasks bridging the gap between discrete and continuous representations. We show that it is possible to express many physical problems in a wavelet framework, thereby allowing the derivation of efficient algorithms that take advantage of wavelet properties, such as multiresolution structure, sparsity, and space-frequency decompositions. We review several examples of such algorithms with applications to X-ray tomography, digital holography, and confocal microscopy and discuss possible future extensions to other modalities.
\end{abstract}

Keywords: Image reconstruction, wavelets, splines, tomography, digital holography, Fresnelets, confocal microscopy.

\section{INTRODUCTION}

Image reconstruction problems involve accurately modeling the physics of the imaging process. This is unlike many general purpose image processing techniques, for example image compression, which are not necessarily bound by such constraints. Yet, many of the latter algorithms' properties, such as speed and robustness, would benefit to image reconstruction techniques. However, their adaptation is not straightforward.

This paper is a brief overview of three image reconstruction techniques that benefit from a multiresolution formalism adapted to match the physical imaging process. The hope is that the underlying methodology will inspire the design of similar algorithms in other fields. Examples are introduced from tomography, ${ }^{1}$ digital holography, ${ }^{2,3}$ and fast confocal microscopy. ${ }^{4}$ The adaptation of the multiresolution formalism and, in particular, the use of wavelets ${ }^{5}$ permits the derivation of flexible and robust algorithms that take advantage of properties such as sparsity and robustness to noise.

\section{TOOLS: B-SPLINES AND WAVELETS}

A continuously defined, finite-energy function can be approximated by a discrete decomposition using various function bases. Possible basis candidates for such decompositions are the complex exponential functions of Fourier series, GaussHermite functions, shifted B-spline or wavelet bases. While the former two bases allow convenient computation in conjunction with physical operators (e.g. complex exponentials are eigenfunctions of convolution operators), the representation of an arbitrary image within such bases requires a high number of coefficients for the image to be satisfactorily approximated. The main reason for this drawback is that the basis functions are not localized in space. B-splines and wavelet bases are localized.

B-splines are piecewise polynomials with a finite support. The B-spline of degree zero is the unit box function. Higher degree B-splines are obtained by recursive convolution of the box function: the B-spline of degree one is a triangle function, the auto-correlation of the box function. A basis can be generated by shifting a B-spline (of fixed degree) on unity spaced nodes on the real axis. Continuous functions can then be approximated by a function that lies in the subspace spanned by the B-spline basis and yield a discrete set of coefficients. Conversely, it is possible to convert a series of discrete samples into a continuous function by computing B-spline coefficients such as to interpolate the samples. ${ }^{6}$ This powerful

M.L.: E-mail: liebling@ caltech.edu, Telephone: +1-626-395-2863

Photons Plus Ultrasound: Imaging and Sensing 2007: The Eighth Conference on Biomedical Thermoacoustics, Optoacoustics, and Acousto-optics, edited by Alexander A. Oraevsky, Lihong V. Wang,

Proc. of SPIE Vol. 6437, 64371C, (2007) - 1605-7422/07/\$18 - doi: 10.1117/12.701352

Proc. of SPIE Vol. 6437 64371C-1 
formalism permits to bridge the gap between discrete measurements and continuously defined operators by transforming the discrete samples into a continuous function.

When B-splines are scaled by a factor of two, yielding functions that have a support twice as large as the original function, and are shifted by steps that are twice that of the original distance between the nodes, this new family may be used to obtain a coarser approximation of the original signal. The coarse approximation requires only half the coefficients of the fine approximation. The whole process can be iterated to generate even coarser representations. Since coarse approximations are in the subspaces spanned by the finer bases, the process of obtaining coarser approximations can be carried out recursively and efficiently. Also, this embeddedness property is central to building wavelet bases. ${ }^{7,8}$

For a given multiresolution approximation, the wavelet basis is obtained from a single function that is scaled and shifted. Starting from a fine approximation of the signal, the wavelet decomposition yields a coarse signal (which is, in the case of B-spline wavelets, equivalent to the coarse approximation described in the previous paragraph) and, instead of the fine representation itself, the difference between the coarse and the fine representation. The number of coarse and detail coefficient is equal to the number of coefficients of the fine representation. For a given scale, the corresponding wavelet functions span the detail space. Wavelets can themselves be built from B-splines ${ }^{9}$ and there exist efficient algorithms to compute the decomposition coefficients. ${ }^{5}$

Simple functions, say, step functions, require many harmonics in order to be represented accurately using a Fourier series. Conversely, using a basis that is localized, such as a wavelet basis, permits to capture locally changing signals using only few coefficients, and therefore represent the signal using a sparse set of coefficients.

\section{APPLICATIONS TO IMAGE RECONSTRUCTION}

\subsection{Continuous Operators and Discrete Samples}

Physical acquisition processes are often described using operators that act upon continuous functions. Examples include the Radon transform for computerized tomography, ${ }^{1}$ the Fresnel transform in digital holography, ${ }^{10}$ or the general form of an optical imaging system modeled by the convolution with a point spread function. ${ }^{11}$ This continuous formalism is in contrast to the experimental measurements that correspond to discrete samples of a continuous signal. Since such operators act on continuous functions, they cannot be used, as is, to process discrete samples. Approximating the operators to a discrete form is a solution that may give satisfactory results in some situations but has the drawback of modifying the operator, thereby possibly introducing errors into the model itself.

Alternatively, the B-spline and wavelet formalism described above permits to retain the continuously defined operators in their pristine form and, via interpolation of the samples, apply it directly to continuous functions. When the operator is linear, it acts on the shifted basis functions of the signal's linear decomposition, leaving the coefficients "intact". In other words, to compute the action of a linear and continuously defined operator on a function described by a linear combination of shifted basis functions, it is sufficient to compute the action of the operator on the generating basis function. Whenever it is possible to obtain a closed form expression for the transform of the elementary building blocks, the transform of the original signal is obtained by replacing the original basis functions by their transforms, yielding straightforward and fast implementations.

In the case of B-splines and wavelets built from B-splines, closed-form expression of their Fresnel transform (Fresnelets ${ }^{12}$ ) and their Radon transform (spline bi-kernels ${ }^{13}$ ) can be derived. The advantage of such an approach over a discretization of the the operator itself is that the approximation error can be controlled and is limited to the interpolation of the discrete samples while the the transform is exact. In tomography, using continuously defined functions in a discrete setting permits the derivation of elegant algorithms. ${ }^{13,14}$

\subsection{Robust Multiresolution Techniques}

The multiresolution formalism comes in handy when solving reconstruction problems that involve finding unknown parameters. The guiding principles of iterative multiresolution algorithms is to solve parametric problems at a coarse resolution, and then iteratively refine the solution by working at a finer resolution. The solution obtained at the coarse resolution level is taken as the initial guess for computing a better solution at a finer level. Thereby, being trapped in local minima can be avoided. This approach has been applied successfully for image registration ${ }^{15}$ or image segmentation using snakes. ${ }^{16}$ Multiresolution reconstruction strategies have also been carried out in tomography, notably for region of interest reconstruction ${ }^{17}$ or in the context of more sophisticated acquisition geometries. ${ }^{18}$ 
Since wavelet decompositions inherently bear a multiresolution structure, it is possible to solve problems in the wavelet domain instead of the space domain with similar advantages. For example, Fresnelets permit reconstructing complex wave fields in digital holography from diffraction patterns. Propagation is modeled by the Fresnel transform, with the propagation distance as its parameter. Changing the propagation distance changes the "focus" of the reconstructed wave-fields. While several methods for measuring the focus of an image exist, methods that are robust to noise and fast are more difficult to derive. Within the Fresnelet formalism, one Fresnelet basis is associated to each propagation distance. A reconstruction at a given distance can be obtained by decomposing the complex wave in a Fresnelet basis of that distance and reconstructing it with a standard wavelet, respectively in a B-spline basis. As it turns out, sparse decompositions yield focused images. Therefore, adjusting the focus is equivalent to finding the Fresnelet basis for which the energy of a set percentage of coefficients is maximum. ${ }^{19}$ Using similar arguments, it is also possible to efficiently compress digital holograms. ${ }^{20}$

Another problem, arising when imaging objects that undergo periodic motion, is the synchronization of several image sequences. If, during the time it takes to acquire an entire three-dimensional volume using a laser scanning confocal microscope, the object undergoes motion, the image data does not give an accurate representation of the object. There is an alternative way of acquiring such dynamic volumes, provided the motion of the object is cyclical and the microscope frame rate allows for the acquisition of image series at a fixed depth in the sample at high speed. ${ }^{4}$ The method consists in successively acquiring image series at increasing depths throughout the sample. The focus is set at a fixed depth and a time-series of the confocal slice is acquired at high speed for 2-4 periods of the object's cyclic motion. Then, the focus is changed to a deeper section. This sequential acquisition procedure is repeated until the whole object has been imaged at all depths. The sections can be assembled provided that all time-series are synchronized. When they are not synchronized, a situation that arises when the acquisition is not gated to the object motion, volumes can still be reconstructed by registering the sequences via the minimization of the intensity difference between slices. ${ }^{21-23}$ The direct minimization in the spatial domain on a pixel by pixel basis is inefficient because the datasets are large and corrupted by noise. The minimization criterion can be translated into the wavelet domain, where it is sufficient to compute the differences on a subset of coefficients (coarse approximation of the image) and where supplementary noise reduction operations can easily be carried out. The resulting algorithm is both robust and fast.

\section{CONCLUSION}

Multiresolution and wavelet approaches to reconstructing images have advantages that can be summarized as follows.

- They provide the necessary formalism to link continuous representations with discrete algorithms.

- Existing wavelet algorithms developed for general image processing purposes can be integrated naturally and seamlessly (compression, noise reduction, etc.)

- Data organization in frequency bands and energy compaction permit data reduction and a sparse representation leading to efficient implementations.

- By operating from coarse to fine resolutions or by taking advantage of multiresolution structure, robust algorithms can be derived.

- The general formalism provides a guiding principle for deriving new algorithms for other problems.

\section{ACKNOWLEDGMENTS}

Michael Liebling is supported by a fellowship from the Swiss National Science Foundation no. PA002-111433.

\section{REFERENCES}

1. A. C. Kak and M. Slaney, Principles of Computerized Tomographic Imaging, IEEE Press, 1988.

2. J. W. Goodman and R. W. Lawrence, "Digital image formation from electronically detected holograms," Appl. Phys. Lett. 11, pp. 77-79, Aug. 1967.

3. L. P. Yaroslavskii and N. S. Merzlyakov, Methods of Digital Holography, Consultants Bureau, New York, 1980.

4. R. Wolleschensky, B. Zimmermann, and M. Kempe, "High speed confocal fluorescence imaging with a novel line scanning microscope," J. Biomed. Opt. 11, p. 064011, Nov.-Dec. 2006. 
5. S. G. Mallat, "A theory for multiresolution signal decomposition-the wavelet representation," IEEE Trans. Pattern Anal. Mach. Intell. 11, pp. 674-693, July 1989.

6. M. Unser, "Splines: a perfect fit for signal processing and image processing," IEEE Signal Process. Magazine 16, pp. 22-38, Nov. 1999.

7. S. Mallat, A wavelet tour of signal processing, Academic Press, 1998.

8. G. Strang and T. Q. Nguyen, Wavelets and Filter Banks, Wellesley-Cambridge Press, Cambridge Mass., 1996.

9. M. Unser, A. Aldroubi, and M. Eden, "A family of polynomial spline wavelet transforms," Signal Processing 30, pp. 141-162, Jan. 1993.

10. J. W. Goodman, Introduction to Fourier Optics, McGraw-Hill, New York, second ed., 1996.

11. T. Wilson, ed., Confocal Microscopy, Academic Press, London, 1990.

12. M. Liebling, T. Blu, and M. Unser, "Fresnelets: new multiresolution wavelet bases for digital holography," IEEE Trans. Image Process. 12, pp. 29-43, Jan. 2003.

13. S. Horbelt, M. Liebling, and M. Unser, "Discretization of the Radon transform and of its inverse by spline convolutions," IEEE Trans. Med. Imag. 21, pp. 363-376, Apr. 2002.

14. K. M. Hanson and G. W. Wecksung, "Local basis-function approach to computed tomography," Appl. Opt. 24, pp. 4028-4039, 1985.

15. P. Thévenaz, U. E. Ruttimann, and M. Unser, “A pyramid approach to subpixel registration based on intensity," IEEE Transactions on Image Processing 7, pp. 27-41, Jan. 1998.

16. P. Brigger, J. Hoeg, and M. Unser, "B-Spline snakes: A flexible tool for parametric contour detection," IEEE Trans. Image Proc. 9, pp. 1484-1496, Sept. 2000.

17. F. Rashid-Farrokhi, K. J. R. Liu, C. A. Berenstein, and D. Walnut, "Wavelet-based multiresolution local tomography," IEEE Trans. Image Proces. 6(10), pp. 1412-1430, 1997.

18. S. Bonnet, F. Peyrin, F. Turjman, and R. Prost, "Multiresolution reconstruction in fan-beam tomography," IEEE Trans. Image Process. 11, pp. 169-176, Mar. 2002.

19. M. Liebling and M. Unser, "Autofocus for digital Fresnel holograms by use of a Fresnelet-sparsity criterion," J. Opt. Soc. Am. A 21, pp. 2424-2430, Dec. 2004.

20. E. Darakis and J. J. Soraghan, "Use of fresnelets for phase-shifting digital hologram compression," IEEE Trans. Image Proces. 15(12), pp. 3804-3811, 2006.

21. M. Liebling, A. S. Forouhar, M. Gharib, S. E. Fraser, and M. E. Dickinson, "Four-dimensional cardiac imaging in living embryos via postacquisition synchronization of nongated slice sequences," J. Biomed. Opt. 10(5), pp. 054001 $1-10,2005$.

22. M. Liebling, A. S. Forouhar, R. Wolleschensky, B. Zimmerman, R. Ankerhold, S. E. Fraser, M. Gharib, and M. E. Dickinson, "Rapid three-dimensional imaging and analysis of the beating embryonic heart reveals functional changes during development," Developmental Dynamics 235, pp. 2940-2948, Nov. 2006.

23. A. S. Forouhar, M. Liebling, A. Hickerson, A. Nasiraei-Moghaddam, H.-J. Tsai, J. R. Hove, S. E. Fraser, M. E. Dickinson, and M. Gharib, "The embryonic vertebrate heart tube is a dynamic suction pump," Science 312, pp. 751753, May 2006. 